\title{
IMPLEMENTATION OF DYNAMIC SOURCE ROUTING (DSR) IN MOBILE AD HOC NETWORK (MANET)
}

\author{
Thiyam Romila Devi ${ }^{1}$, Rameswari Biswal ${ }^{2}$, Vikram Kumar ${ }^{3}$, Abhishek Jena ${ }^{4}$ \\ 1, 2, 3, ${ }_{4}$ M.Tech, School of Electronics, KIIT University, Odisha, India \\ romilath@gmail.com,rameswaribiswal@gmail.com,vikram_2006be@yahoo.co.in,bapuni207@gmail.com
}

\begin{abstract}
Generally, the Base station (BS) is fixed in the mobile communication system however in Mobile Ad hoc network, Base Station (BS)s is not used, thus it is named as infrastructure less network which can manage its network independently. The Routing protocol plays an important role and a lot of research has been done in this area. This paper presents a protocol for routing mobile ad hoc network (MANET) that uses dynamic source routing. This protocol adapts quickly to the change in the routing path as the host is changing its location frequently. DSR allows the network to be fully self-organising and self-configuring without the need for any existing network. The protocol is composed of the two mechanisms of route discovery and route maintenance work together to search for the shortest path and also to maintain source route to arbitrary destination in the Mobile ad hoc network, we used optimize routing protocol in Mobile ad hoc network (MANET).This optimization is done on DSR (Dynamic Source Routing) protocol using ANT algorithm. The performance of DSR is analyzed using Qualnet 5.2 with respect to QOS (Quality of Service) of throughput, end to end delay, Routing overhead, Average hop count. All the parameter are analyzed in three different scenario of velocity, pause time and traffic connection. All the result are compared with the result of standard DSR routing protocol. The comparison shows that the DSR-ant has better performance than standard DSR. By using Ant algorithm, the DSR optimization can be improved with a smaller delay and the number of hop to transfer information between nodes.
\end{abstract}

Keywords: MANET, DSR, ANT Algorithm

\section{INTRODUCTION}

\subsection{MANET Overview}

Mobile ad hoc network (MANET) is a autonomous and dynamic network consist of wireless mobile hosts or nodes. For example, communication between remote notes is based on multiple hop. These nodes are dynamically and arbitrarily located in such a manner that the interconnections between nodes are capable of changing on a continual basis. Thus MANETs are self configuring as there is no central management system with configuration responsibilities. All the mobile nodes can communicate each other directly if they are in others wireless links radio range. In order to enable data transfer they either communicate through single hop or multi hop with the help of intermediate nodes. MANETs allow ubiquitous service access anywhere, anytime without any fixed infrastructure, thus named as an infrastructure-less network that can be widely used in military, battlefields, management services, classrooms, various multimedia applications and conference halls. The Fig-1. shows the MANET

*** $*$

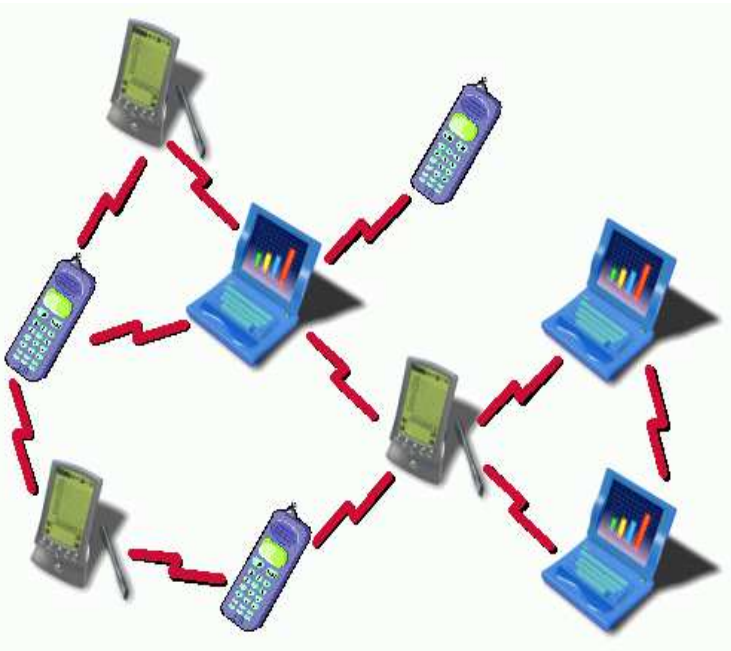

Fig-1: Infrastructure less Network

\subsection{DSR Protocol and Optimization}

The DSR (Dynamic source routing) protocol is a simple and efficient routing protocol for wireless mesh network which is designed for the use in multi- hop wireless ad hoc network of mobile nodes. DSR allows the network to be completely self- 
organizing and self-configuring, without the need of any predefined infrastructure. This protocol is composed of the two main mechanisms of a) Route discovery and b) Route maintenance. These two work together to allow nodes to discover and maintain routes to arbitrarily destination in the ad hoc network [1].

\subsubsection{Route Discovery}

Route Discovery is used to discover a route from source to particular destination using two stages; Route request (RREQ) and Route Reply(RREP). This method also includes some optimization measures; during route request process, and intermediate node can be authorized to issue a complete route reply if it contains a valid route to the destination in its route cache memory. Whenever a source wants to communicate with destination and if it does not have a route in its Route Cache, it broadcasts a RREQ message to find a particular route. Each neighbour receives the RREQ and appends its own address to the address list in the RREQ (if it has not already processed the same request earlier) and re-broadcasts the packet. This process will continue until either the maximum hop counter is incremented (or RREQ is rejected) or the destination is reached. The destination receives the RREQ, appends its address and generates a route reply packet (RREP) back towards the source using the reverse of the accumulated route in latter case. When the source finally receives RREP, it will store the route in its Route Cache [2].

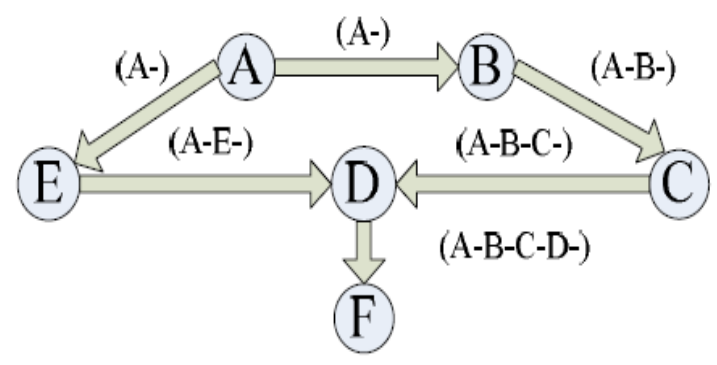

Fig-2: DSR Route Discovery

During the process of discovery, there's a problem that should be noticed. In the Figure 1 above, node $\mathrm{D}$ may receive the router requests both from node $\mathrm{C}$ and node $\mathrm{E}$ at the same time, this will lead to the message collision, and the correct requests cannot be accepted. Therefore, the broadcast in Ad hoc network isn't reliable. This kind of problem can be avoided by random delay transmission, authentication and so on. However, random delay transmission cannot guarantee to choose the best suitable router item, while the authentication has to send more router control messages, thus results in more resource wasted and load aggravation. There comes up a new algorithm to solve this problem, i.e. ANT Algorithm

\subsubsection{Route Maintenance}

Route Maintenance is used to manage (cache, expire, switch among) previously discovered routes. Once some nodes find the neighbouring link that data is to be sent by is disconnected, they immediately send a route error message RERR to source node. When the source node receives the error packet, it deletes all the routes that use the invalid link from the buffer, and starts a new route discovery process if necessary. The nodes that forward the error packet along the way delete all the route in the broken link from their own routing table. The route discovery procedure of DSR protocol often discovers many routes from source node to destination node. And route with minimal hop is more possible chosen for data transmission than others, the nodes frequently chosen are more likely to consume more energy, which results in short usage of battery.

\subsection{Architecture of Mobile communication system}

\section{based on DSR}

DSR is used to replace the former routing protocol in BS. In the same time the backbone network of mobile communication system becomes an entire IP network. Every BS has assigned a unique IP address and the data transmitted between the BSs results into the IP formation of the same data. The Fig- 2. Shows BS of Mobile communication network after the improvement.

The basic elements of BS are-

a) Wireless link control layer- This layer defines reliable data transmission between MS and BS in circuit mode and it can be expanded like as link layer.

b) Wireless resource management layer- It both builds and removes the wireless link between MS and BS for the MS where it moves in network when the link state transmission is being maintained.

c) Mobility management layer- It realizes some functions of MS such as user's register and authorization in the service area of different BSs.

d) Communication management layer- It realizes the functions of management to wireless users such as routing management and routing management.

e) Switching- It realizes the IP's disposal of data. It can be discovered and maintained the route between BSs of source MS and destination MS according to DSR protocol.

f) Wireless channels (Uu) interface- It defines the wireless interface between BS and Ms.

g). Wireless channels (lu) interface- It defines the wireless interface between BSs.

The Fig-3 shows the data flow from one BS to another BS with two wireless hops in mobile communication with the entire mobility of data and also shows how the inclusion of more intermediate BSs would behave. The MS originates a typical data packet and is arriving at the BS A through Uu 
interface. At BS A the packet must transverse the entire protocol stacks, from the Uu interface down to the lu interface. At the intermediate BS, the packet must brought up to DSR sub layer and will be examined and is sent out again. Hence, packets do not go beyond the DSR sub layer in the route. All the packets are originated from and is terminated at the sub layer of DSR. The relaying of control packets is same as that of the data packets.

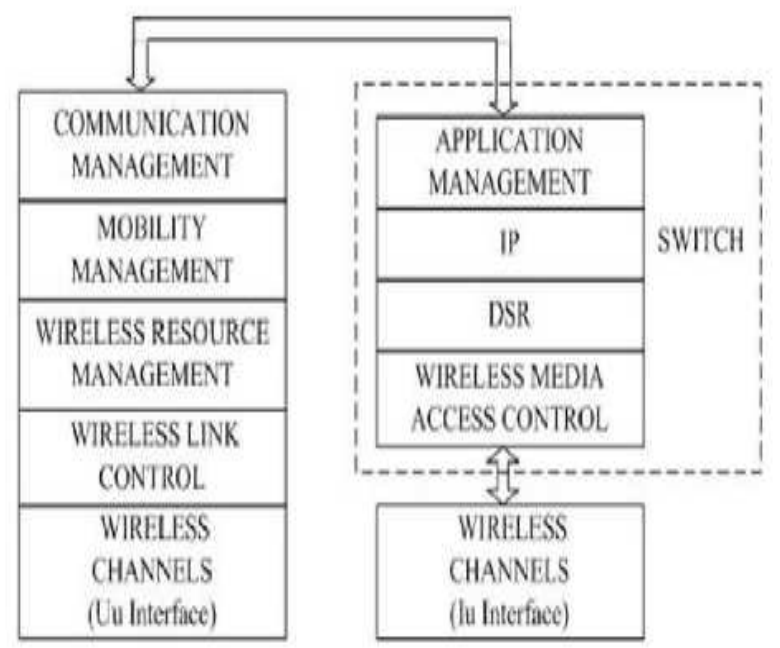

Fig-3: BS of Mobile Communication Network

\section{ANT ALGORITHM}

ANT algorithm is an algorithm that is similar as the behavior of ant colonies, known as ant system. Ant can find the shortest route between the nest and the food source by footprints or pheromones on the path that has been traversed. Ants are practically blind but they still manage to find their way to and from food. These observations inspired a new type of algorithm called ant algorithms (or ant systems)[3][4][5][6]. In order to determine the shortest route, Ant algorithm is being used and the steps of determining are as follows:-

a). Initialization parameters value algorithm. The intensity of inter-node ant trail and changes ( $\tau$ ij), many of nodes (n) includes the coordinates $(\mathrm{x}, \mathrm{y})$ or the distance between nodes (dij), Origin node and destination node, constant cycle of ants $(\mathrm{Q})$, constant intensity controller ant trail $(\alpha)$, the value of $\alpha \geq 0$, constant control visibility $(\beta)$, the value of $\beta \geq 0$, visibility between nodes $=1 / \mathrm{dij}$ ( $\eta \mathrm{ij}$ ), the number of ants $(\mathrm{m})$, evaporation constant trail of ants $(\rho)$, $\rho$ values must be $>0$ and $<1$ to prevent infinite pheromone trail, and the maximum number of cycles (NCmax) is fixed during the algorithm run, while costs
( $\tau$ ij) will be updated at each cycle algorithm from the first cycle $(\mathrm{NC}=1)$ to reach the maximum number of cycles $(\mathrm{NC}=\mathrm{NCmax})$ or until there is convergence. After we initialization parameters, further we Initialize the first node each ant. First performed initialization ( $\tau$ ij), then (m) ants are placed on certain first node randomly. This ant called forward ant packet, it will trace the route to the destination node. When it was discovered the destination node, the ants would go back through the same route. In this condition the ant called backward ant.

b). Charging the first node in the tabu list. Tabu list is a table containing the visibility of information between nodes. The results of the first node initialization every ant in first step should be filled as the first element of the table list. The result of this step is to fill its first element table list each ant with a particular node index, which means that every $\operatorname{tabu}_{\mathrm{k}}$ (1) can contain node index between 1 and $\mathrm{n}$ as the initialization in step (a).

c). Composition of each ant route to all node. Ant colonies that have been distributed to some or each node will start to travel from each first node as the origin node and one other node as the destination node. Then from the second node, respectively, ant colonies will continue the trip by choosing one of the nodes that are not on $\operatorname{tabu}_{\mathrm{k}}$ as the next destination node. Journey ant colony continues until all the nodes one by one visited or have occupied tabu $\mathrm{k}_{\mathrm{k}}$.If $\mathrm{s}$ expressed sequence index visit, expressed as tabu $\mathrm{u}_{\mathrm{k}}$ origin node (s) and other node is expressed as $\left\{\mathrm{Ntabu}_{\mathrm{k}}\right\}$, then the destination node is used to determine the probability of the node equations to visit [3][4][5][6]:

$$
P_{i j}^{k}=\frac{\left[\tau_{i j}\right] \alpha *\left[\eta_{i j}\right] \beta}{\sum\left[\tau_{i k \prime}\right] \alpha *\left[\eta_{i k \prime}\right] \beta}
$$

for $\mathrm{j} \in\left\{N-\operatorname{cabu}_{k}\right\}$ arcd $P_{i j}^{k}=0$, for other $\mathrm{j}$ with $\mathrm{I}$ as the index of the origin node and $\mathrm{J}$ as index destination node.

d). Calculation of route length of every ant. Calculation of closed route length (length closed tour) or $\mathrm{Lk}$ any ants performed after one cycle completed by all ants. This calculation is done based each tabu $\mathrm{k}_{\mathrm{k}}$ with the following equation with dij is the distance between node $i$ to node $j$ $[3][4][5][6]$ :

$$
\begin{aligned}
& \mathrm{L}_{\mathrm{k}}=d_{\text {tabus }}(n), \operatorname{tabu}_{k}(1)+\sum_{s=1}^{n-1} \mathrm{~d}_{\mathrm{tabu}_{\mathrm{k}}}(\mathrm{s}), \operatorname{tabu}_{\mathrm{k}}(\mathrm{s}+1) \\
& d_{i j}=\sqrt{\left(\mathrm{x}_{1}-\mathrm{y}_{\mathrm{j}}\right)^{2}+\left(\mathrm{y}_{1}-\mathrm{y}_{\mathrm{j}}\right)^{2}}
\end{aligned}
$$


to search the shortest route. After every ant $\mathrm{Lk}$ is calculated, will get a minimum length of each cycle routes closed or Lmin NC and minimum values on the whole length of the route is closed or Lmin. Calculation of changes in the value of the intensity of inter-node ant footprints. Ant colonies will leave footprints on the path between the nodes in its path. The existence of evaporation and the difference in the number of ants making the possibility of a change in the value of the intensity of inter-node ant footprints. The equation this changes is $[3][4][5][6]$ :

$$
\Lambda \tau_{i j}=\sum_{l=1}^{m} A \tau_{i j}^{k}
$$

$\Delta \tau_{i j}^{k}$

Changes in intensity values between nodes and footprints every ant is calculated based on the equation:

$\Delta \tau_{i j}^{k}=\frac{Q}{L_{k}}$, for $(\mathrm{i}, \mathrm{j}) \in$ origin node and destination node in $\mathrm{tabu}_{\mathrm{k}}$

$=0$, for other $(\mathrm{i}, \mathrm{j})$

e). The calculation of the value of the intensity of internodes ant footprints for the next cycle. Ant footprints intensity values between nodes on all paths between nodes is likely to change due to evaporation and the difference in the number of ants that passed. For the next cycle, the ant will pass the track intensity prices have changed. Ant footprints intensity values between nodes for the next cycle is calculated by the equation [3][4][5][6]:

$$
\tau_{i j}=\mathrm{P}^{*} \tau_{i j}+\Delta \tau_{i j}
$$

further Reset the value of the intensity changes between nodes ant footprints. For the next cycle of price changes ant trail intensity between the nodes need to be rearranged in order to have a value equal to zero.

f). Emptying of tabu list, and repeat step b if necessary. Tabu list needs to be emptied to be filled again with the new order of nodes in the next cycle, if the maximum number of cycles has not been achieved or has not convergence. Algorithm is repeated from step $b$ with prices intensity parameter ant footprints among nodes that have been updated.

\section{SIMULATION}

\subsection{Simulation Parameter}

In this paper we simulate the transmission of packet data in mobile Ad Hoc network using DSR protocol that has been optimized using Ant algorithm ( DSR-Ant). For simulation we have used Qualnet 5.2 Software. In our simulation we have taken simulation area $500 \times 500$ meters, radio propagation model Rayleigh and Rician and simulation time $100 \mathrm{~ms}$. The performance will be measured in terms of Qos parameters of delay and throughput. For analyzing routing scenario, we have considered routing overhead and hop count. Above mentioned parameters plays very important roles in analyzing the network. Delay and throughput parameter gives performance at receiver side and routing overhead and hop count parameter used to analyze routing performance based on algorithm used.

\subsection{Scenario Analysis}

We have analyzed three scenarios to evaluate DSR performance in velocity of node movement. This is to evaluate the routing protocol performance in velocity variation and environment.

- In first scenario, we have fixed nodes and increasing the velocity of node and data is being transmitted between the nodes. In this we used 9 values between $2 \mathrm{~m} / \mathrm{s}$ to $18 \mathrm{~m} / \mathrm{s}$.

- In second scenario, we implement various pause time to evaluate routing protocol performance in dynamic network. We have used pause time values between 10 s to 100 s.

- In third scenario, we have implemented constant velocity with increasing traffic connection between the node. We have used ten different traffic connection and the number of node between 2 to 20 . When there is more traffic then queuing is higher and produces congestion in network.

\section{RESULT AND ANALYSIS}

\subsection{For velocity scenario}

The result shows higher throughput and smaller end -to-end delay as shown in Fig-4.1(a) and Fig-4.1(b)[7]. DSR-ant searches the best path needs more routing overhead in a manner such that protocol find shortest path with smaller hop change in velocity will effect the shape and configuration of network data transmission path. In this scenario, we have analyzed that DSR-ant performance is better than standard DSR in throughput, smaller end-to-end delay and number of hop to send the message but DSR-ant has larger routing overhead. 


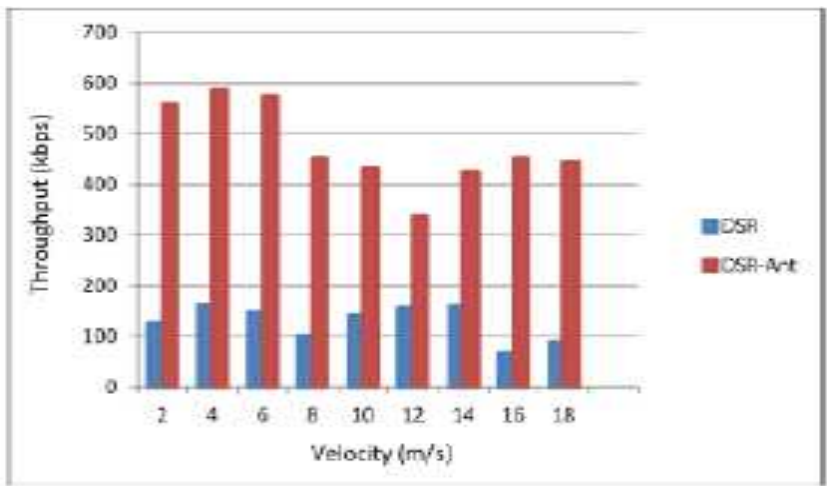

Fig-4.1: (a) Throughput

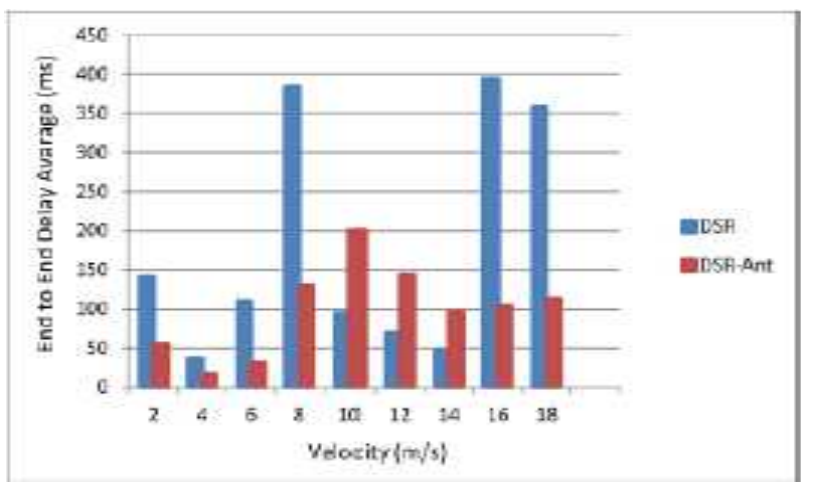

Fig-4.1: (b) End-to-end delay

\subsection{Result and Analysis Pause Time Scenario}

The Fig-4.2(a) and Fig-4.2(b)[7] shows the pause time scenario of end-to-end delay and Throughput. We have analyzed that DSR-ant has smaller end-to-end delay because this algorithm can search most efficient path with higher throughput than standard DSR.

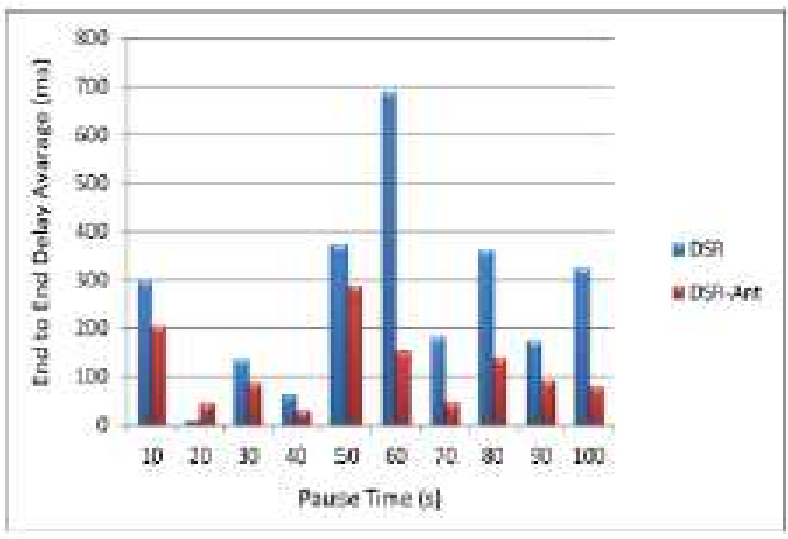

Fig-4.2: (a) End-to-end delay

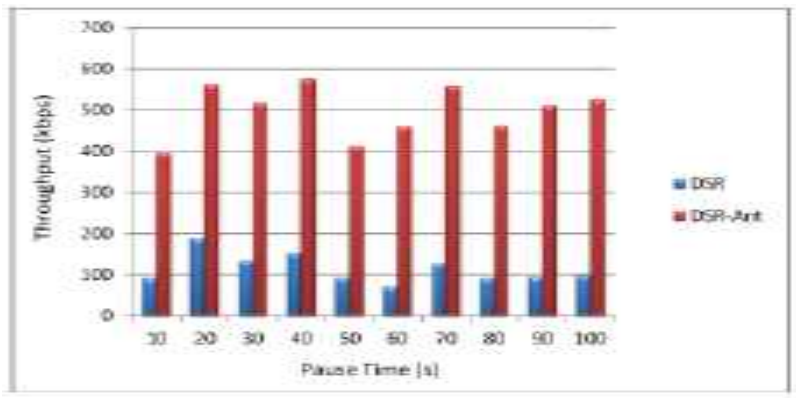

Fig-4.2: (b) Throughput

\subsection{Result and Analysis Traffic Connection Scenario}

The traffic connection scenario is shown in Fig-4.3 (a), (b), (c) and (d)[7]. When we are increasing traffic connection in the network between the user or node will create congestion, larger number of packets will wait in queue in the system and lesser bandwidth. The routing protocol that has been optimized with Ant algorithm can improved the performance with smaller end-to-end delay and lesser number of hop. DSRant have higher throughput in comparison to standard DSR but due to complex calculation in DSR-ant to find the shortest path, we have higher value of routing overhead.

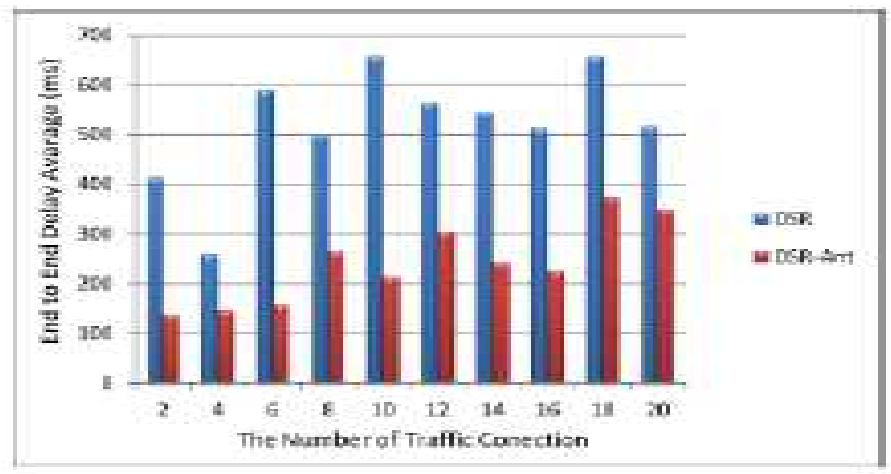

Fig-4.3: (a) End-to-end delay

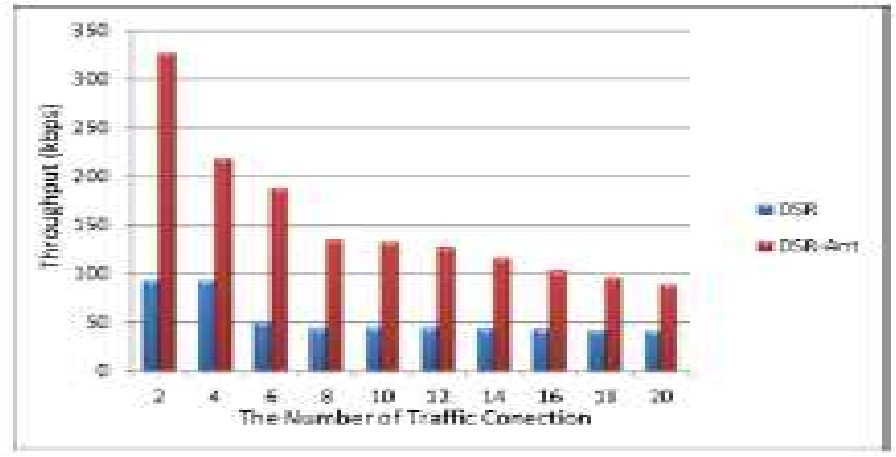

Fig-4.3: (b) Throughput 


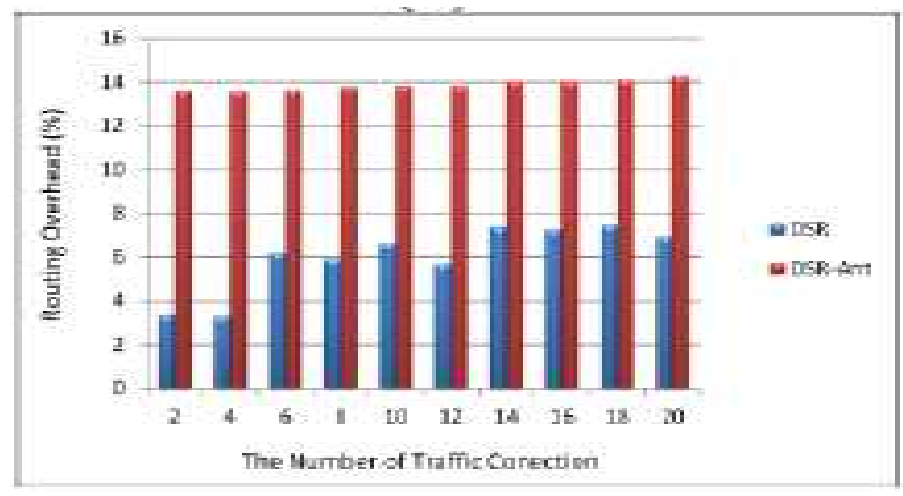

Fig-4.3: (c) Routing overhead
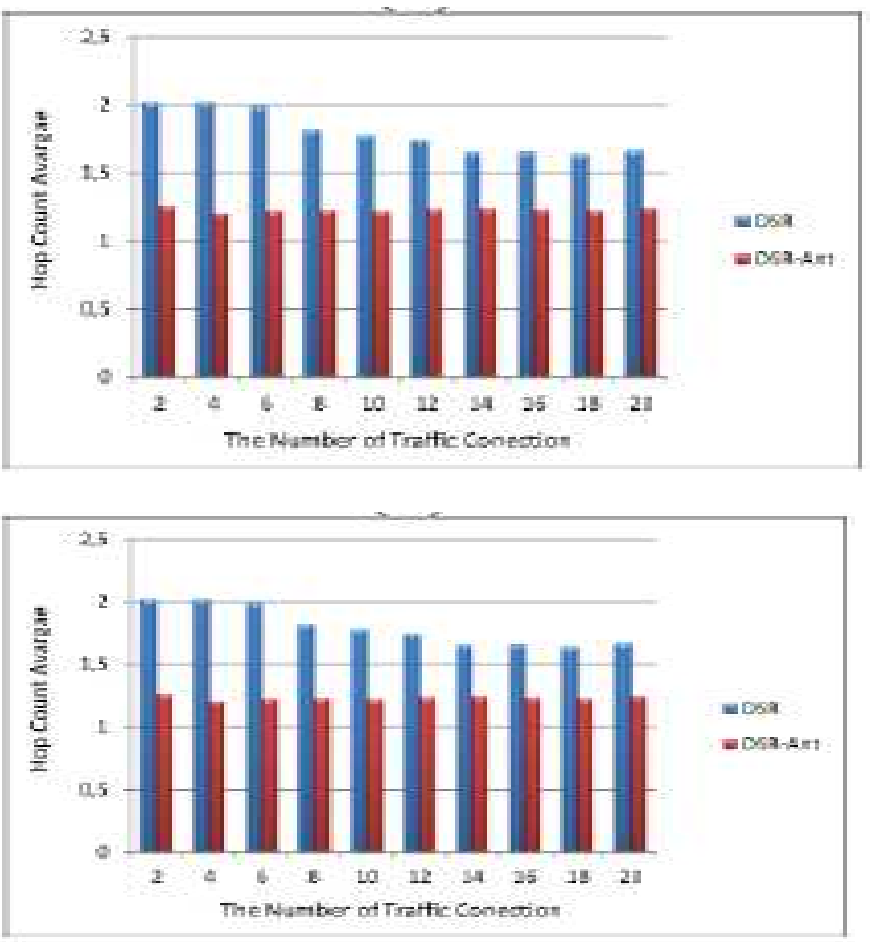

Fig-4.3: (d) Average hop count

\section{CONCLUSIONS}

Ant algorithm is a very good tool to be implemented in optimization of mobile ad hoc network. we have implemented it using Qualnet 5.2 in reference to three different scenario and compared our result with a standard DSR. Our result shows that DSR-ant can improve the performance of MANET in respect to end-to-end delay, throughput and number of hop to transfer the message from sender to receiver. We can reduce time needed to search for the shortest path using the Ant algorithm. This will makes our mobile Ad hoc network faster

\section{REFERENCES}

[1]. Istikmal, Meylanie Olivya, "Performance Analysis of Routing Protocol on Data Forwarding Effectiveness in Mobile Ad hoc Network", Proceedings of The 5th International Conference on Telematics System, Services and Applications. Institute Technology of Bandung, November 19-21, 2009.

[2]. Zhaohua Long, Zheng He."Optimization and Implementation of DSR Route Protocol based on Ad hoc network".

[3]. Gianni Di Caro and Marco Dorigo. "AntNet: Distributed Stigmergetic Control for Communications Networks". Journal of Artificial Intelligence Research, 9:317-365, 1998.

[4]. Gianni Di Caro."Ant Colony Optimization and Its Application to Adaptive Routing in Telecommunication Network"

[5]. Vincensius LR, Indrarini Dyah I, Istikmal . "Performance Analysis DSDV and ZRP based Ant algorithm at Mobile Ad hoc network". Telecommunications Journal of Research and Development, december 2010 volume 15-number 2,pp-109 [6]. Leksono, Agus."Ant Colony Optimization (ACO) Algorithm to Completed Travelling Salesman Problem (TSP). Faculty of Mathematics and natural Sciences, University of Dipengoro,2009.

[7]. Istikmal."Analysis And Evaluation Optimization Dynamic Source Routing ( DSR ) Protocol in Mobile Adhoc Network Based on Ant Algorithm". International Conference of information and communication technology (ICoICT),2013.

\section{BIOGRAPHIES}

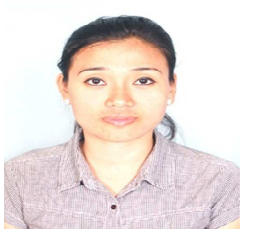

Thiyam Romila Devi:- Has received B.E in Electronics and communication Engineering from KNSIT, Bangalore under Visvesvaraya Technological University (VTU),Belgaum, India. She is presently pursuing $\mathrm{M}$. Tech from KIITUniversity, Bhubaneswar, Odisha, India with specialization in Communication System Engineering (ETC).

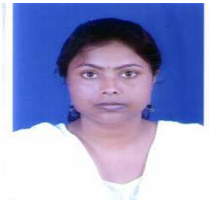

Rameswari Biswal: Has received B.Tech in Electronics and Communication Engineering from BCET, Balasore under Biju Patnayak University of Technology (BPUT), India. She is presently pursuing $M$. Tech from KIIT-University, Bhubaneswar, Odisha, with specialization in Communication System Engineering (ETC).

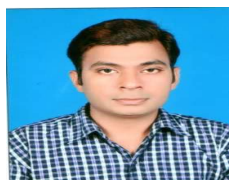

Vikram Kumar:- Has received B.E. in Electronics and communication Engineering from LNCT, Bhopal under Rajiv Gandhi Proudyogiki vishwavidyalaya (RGTU), India. He is presently pursuing M. Tech from KIIT-University, Bhubaneswar, Odisha, with specialization in Communication System Engineering (ETC). 
Abhishek Jena:- Has received B. Tech in

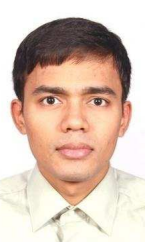

Electronics and communication

Engineering from SRM College of

Engineering ,Chennai under SRM

University, India. $\mathrm{He}$ is presently

pursuing M. Tech from KIIT-University,

Bhubaneswar, Odisha, with specialization

in Communication System Engineering (ETC) 